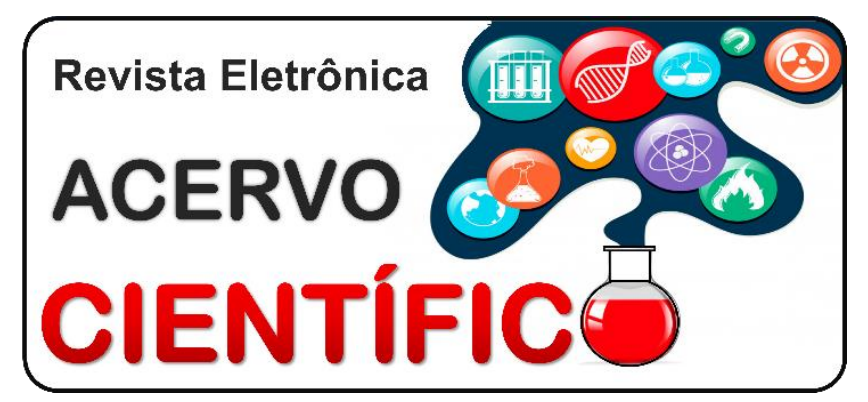

REVISÃO BIBLIOGRÁFICA

Recebido em: 9/2020

Aceito em: 9/2020

Publicado em: 10/2020

\title{
Diagnóstico, tratamento e seguimento da mola hidatiforme: uma revisão bibliográfica
}

\author{
Diagnosis, treatment and follow-up of hydatiform mole: a bibliographic review
}

\section{Diagnóstico, tratamiento y seguimiento de la mola hidatiforme: una revisión bibliográfica}

Ana Carolina Gomes Barroso Ferreira Mattos ${ }^{1 *}$, Danielle Fiorin Ferrari Novais ${ }^{1}$, Gabriela Bahia Ribeiro Reis ${ }^{1}$, Guilherme Ribeiro da Silva Rocha ${ }^{1}$, Matheus Coelho Prudêncio ${ }^{1}$, Velsa Correia da Silva Reis ${ }^{1}$.

\begin{abstract}
Resumo: Esse artigo buscou analisar, por meio de uma revisão de literatura, a produção científica acerca da abordagem aos pacientes com doença trofoblástica gestacional (DTG), a fim de discutir os conhecimentos atuais sobre seu diagnóstico e tratamento. As DTGs constituem-se um grupo de tumores relacionados à gestação, e que apresentam em comum, uma hiperproliferação trofoblástica. Dentre as DTGs, a condição mais frequentemente observada é a mola hidatiforme $(\mathrm{MH})$. $\mathrm{A} \mathrm{MH}$ apresenta desafios para a realização do diagnóstico precoce, tratamento imediato, controle de complicações iniciais e tardias, e seguimento pós-molar adequado. O diagnóstico na maioria das vezes é feito através do exame clínico, da dosagem do hormônio beta hCG e da avaliação ultrassonográfica, mas em alguns casos pode ser necessário o exame histopatológico, considerado padrão ouro. É fundamental no segmento pós gestação molar, uma anticoncepção eficaz e avaliação do risco de transformação maligna, se fazendo de extrema importância no segmento das pacientes com mola hidatiforme, identificar aquelas que apresentam persistência da doença.
\end{abstract}

Palavras-chave: Gonadotrofina coriônica, Monitoração fetal, Mola hidatiforme.

\begin{abstract}
The present study sought to analyze, through a literature review, the scientific production on patients diagnosed with gestational trophoblastic disease (GTD), to discuss current knowledge about its diagnosis and treatment. GTDs defines a spectrum of tumors related to pregnancy, that presents with a hyperproliferation of trophoblastic tissue. Regarding to the GTDs, the most frequent condition is the hydatiform mole (HM). HM presents challenges facing early diagnosis, immediate treatment, and a adequate follow-up to avoid early and late complications. The diagnosis is most often made through clinical examination, beta hCG hormone measurement and US evaluation, but in some cases, histopathological examination, considered the gold standard, may be necessary. It is essential in the post-molar pregnancy segment, effective contraception and risk assessment of malignant transformation, becoming extremely important in the segment of patients with hydatidiform mole, identifying those who have persistent disease.
\end{abstract}

Keywords: Chorionic gonadotropin, Fetal monitoring, Hydatidiform mole.

\footnotetext{
1 Universidade José do Rosário Vellano (UNIFENAS), Belo Horizonte - MG.

*E-mail: anacarolinagbfm@hotmail.com
} 
Resume: Este artículo buscó analizar, a través de una revisión de la literatura, la producción científica sobre el abordaje de pacientes con enfermedad trofoblástica gestacional (EGD), con el fin de discutir los conocimientos actuales sobre su diagnóstico y tratamiento. Los DTG son un grupo de tumores relacionados con el embarazo, que tienen en común una hiperproliferación trofoblástica. Entre los DTG, la afección observada con mayor frecuencia es la mola hidatiforme (MH). La HM presenta desafíos para el diagnóstico precoz, el tratamiento inmediato, el control de las complicaciones tempranas y tardías y el seguimiento posmolar adecuado. El diagnóstico se realiza con mayor frecuencia mediante examen clínico, medición de la hormona beta hCG y evaluación ecográfica, pero en algunos casos, puede ser necesario un examen histopatológico, considerado el estándar de oro. Es fundamental en el segmento de gestación postmolar, la anticoncepción eficaz y la evaluación del riesgo de transformación maligna, siendo de suma importancia en el segmento de pacientes con mola hidatiforme, identificando aquellas que presentan enfermedad persistente.

Palabras clave: Gonadotropina coriónica, Monitoreo fetal, Mola hidatiforme.

\section{INTRODUÇÃO}

A gravidez molar e neoplasias trofoblásticas gestacionais originadas do tecido placentário são raros tumores humanos, que podem dar origem a metástases, mas que em geral são plenamente curáveis. A doença trofoblástica gestacional (DTG) constitui um grupo de tumores relacionados à gestação e que possuem em comum, a proliferação excessiva do tecido trofoblástico, cujo marcador biológico-hormonal é a gonadotrofina coriônica humana (hCG) (DELMANTO LRMG, et al., 2007). Dentre as DTGs, a condição mais frequente é a mola hidatiforme $(\mathrm{MH})$, que se caracteriza por hipertrofia trofoblástica e edema de vilosidades coriônicas, representando 90\% dos casos de DTG. (RAMÍREZ CAG, et al., 2018).

A DTG é uma anomalia que afeta 1 em cada 1000 gestações (SUKSAI M, et al., 2017). Em mulheres brasileiras pode ocorrer 1 em cada 200 a 400 gestações, incidência 5 a 10 vezes maior que a encontrada nos Estados Unidos da América e Europa (LÓPEZ CL, et al., 2018). É mais comum nos extremos da idade reprodutiva, em adolescentes e em pessoas acima de 35 anos de idade. Mulheres com história de gravidez molar tem risco aumentado de recorrência comparado com aquelas sem história prévia. (ABREU EMF, et al., 2009). Apesar da maioria das mulheres portadoras de Mola Hidatiforme ter futuro reprodutivo normal, o risco de nova gestação molar aumenta em até cinco vezes após a primeira gravidez molar (ANDRADE JM, et al., 2009).

A DTG tem como o sintoma mais frequente o sangramento transvaginal, podendo manifestar também hiperêmese gravídica, pré-eclâmpsia precoce, útero aumentado para a idade gestacional, hipertireoidismo e cistos teca-luteínicos ovarianos. A classificação atual da DTG, reconhecida pela Federação Internacional de Ginecologia e Obstetrícia (FIGO) inclui formas pré-malignas, sendo a mola completa e mola parcial e formas malignas, destacando as neoplasias trofoblásticas gestacionais (NTG), que por exame anatomopatológico distinguem-se entre mola invasora, coriocarcinoma e tumor trofoblástico do sítio placentário (MAESTÁ I, BRAGA A, 2012). A forma maligna da doença trofoblástica é a neoplasia trofoblástica gestacional (NTG) , que é definida pela persistência de tecido trofoblástico ativo na parede uterina, podendo ser encontrada em outros órgãos e tecidos. O diagnóstico precoce da forma maligna, com um tratamento adequado, caracteriza a cura da doença e resulta na eficácia da capacidade reprodutiva (DELMANTO LRMG, et al., 2007).

O diagnóstico possui três pilares: o exame físico minucioso, detalhando os principais sinais e sintomas do agravo, os exames laboratoriais, sendo o principal a dosagem do hormônio gonadotrofina coriônica humana e o exame ultrassonográfico (SOARES BC, et al., 2011). O hormônio gonadotrofina coriônica humana (hCG) além de ser utilizado para diagnóstico, ele também é um marcador biológico de relevância no seguimento da doença. Ele é um indicador sensível de doença persistente, podendo ter valores aumentados mesmo após o término do tratamento indicado (LAVIE I, et al., 2005).

Esse artigo buscou analisar, por meio de uma revisão narrativa da literatura, a produção científica acerca da abordagem aos pacientes com mola hidatiforme, a fim de discutir os conhecimentos atuais sobre seu diagnóstico e tratamento. 


\section{REVISÃO BIBLIOGRÁFICA}

\section{Definição e classificação da Doença Trofoblástica Gestacional}

A MH pode ser dividida em MH completa (MHC) e em MH parcial (MHP) de acordo com as diferenças quanto aos aspectos morfológicos (macroscópicos), histopatologia e cariótipo. A MHC se encontra, à macroscopia, com vesículas placentária em seu todo, não encontrando os anexos e concepto. Os cachos de uva, caraterizadas como as vesículas, são integradas de líquido claro, com diâmetro de 1 a 1,5 mm no primeiro trimestre e de 1,5 a $3 \mathrm{~cm}$ no segundo trimestre. Já a MHP está presente vesículas hidatiformes na placenta, associada ao concepto ou anexos fetais. As vesículas são pequenas $(5 \mathrm{~mm}$ no primeiro trimestre até $2 \mathrm{~cm}$ no segundo trimestre) e pouco hidrópicas com área de vilosidades normais (BRAGA A, et al., 2014).

Em relação à histopatologia, as $\mathrm{MHC}$ são caracterizadas por não possuírem elementos fetais, mostram proliferação generalizada e mais significativa do trofoblasto e maior frequência de anaplasia celular. Quanto ao cariótipo, a MHC é o resultado da fecundação de um óvulo sem núcleo ativo, o que significa que todos os genes na MHC são de origem paterna. Entre as MHC, 90\% têm cariótipo 46, XX e 10\%, 46, XY. Já a MHP ocorre quando um óvulo normal é fecundado por dois espermatozóides ou um espermatozóide diplóide, resultando em uma anomalia associada ou causada, mais frequentemente, por triploidia (69XXY) (ANDRADE JM, et al., 2009; TROMMEL NEV, et al., 2005).

Do ponto de vista clínico, em geral, pacientes portadoras de MH podem apresentar volume uterino aumentado para a idade gestacional, hiperemese, hipertireoidismo, sangramento transvaginal de repetição, eliminação de vesículas hidrópicas, pré-eclâmpsia e sintomas consistentes com abortamento incompleto ou retido. Complicações clínicas graves ocorrem em mais de $25 \%$ das pacientes com gravidez molar avançada, mas são raras entre 10-12 semanas de gestação. Dentre essas complicações, podemos destacar: a mola invasora e complicações pulmonares (CORRÊA IB, et al., 2018).

A doença tem como principais fatores de riscos relatados a história prévia de doença trofoblástica gestacional e a idade materna, sendo que o risco está significativamente aumentado em mulheres com idade acima de 40 anos (BRAGA A, et al., 2018). A história pregressa de aborto espontâneo e os níveis séricos elevados da gonadotrofina coriônica humana, produzido em excesso pelo trofoblasto hiperplasiado, também se enquadram nos riscos (BISCARO A, et al., 2012).

\section{Diagnóstico da Doença Trofoblástica Gestacional}

A suspeição diagnóstica da $\mathrm{MH}$ ocorre geralmente no primeiro trimestre de gravidez, através do exame clínico, e pode ser firmado por meio de exames complementares, sendo o diagnóstico definitivo e padrão ouro a análise histopatológica do material obtido através do esvaziamento uterino (HAHN L, et al., 2018).

$\mathrm{Na}$ anamnese e no exame físico podem ser notadas situações suspeitas, dentre elas sangramento, presente em $75 \%$ a $95 \%$ das pacientes, habitualmente indolor, que se inicia, em geral, entre a quarta e a décima sexta semana de amenorreia. Também podem ser observados útero aumentado para a idade gestacional, com altura excedendo em 4 centímetros ao tamanho esperado para a idade gestacional, massas pélvicas anexiais, vômitos incoercíveis e pré-eclâmpsia precoce (antes da vigésima semana de gestação). A eliminação de vesículas é incomum, mas, quando ocorre, pode-se firmar o diagnóstico. Algumas pacientes podem ser assintomáticas. (BRAGA A, et al., 2014).

Diante da hipótese diagnóstica de MH, a FIGO (Federação Internacional de Ginecologia e Obstetrícia) recomenda a dosagem do hormônio Beta-hCG, um marcador biológico de relevância no diagnóstico da doença e no seguimento das pacientes. (KERKMEIJER LGW, et al., 2009). A gonadotrofina coriônica humana é um hormônio glicoproteico, com duas subunidades (alfa e beta), de alto peso molecular, produzida pelo tecido trofoblástico normal e tumoral (CORRÊA IB, et al., 2018). A subunidade alfa é homóloga a subunidade dos hormônios folículo estimulante (FSH), luteinizante (LH) e estimulante da tireoide (THS). Isso pode acontecer reação cruzada com testes pouco precisos e manifestações clínicas acrescidas de reação cruzada. O LH e o FSH poderão aumentar a policistose ovariana, e o TSH, o hipertireoidismo (BRAGA A, et al., 2018). A dosagem de Beta-hCG mostra valores elevados, frequentemente maiores que $100.000,00 \mathrm{mUl} / \mathrm{mL}$, muitas vezes não compatível com a idade gestacional, auxiliando no diagnóstico (BRAGA A, et al., 2014). 
Além do beta-hCG, outro exame de que se tornou aliado importante no diagnóstico de $\mathrm{MH}$ foi a ultrassonografia. A Mola Hidatiforme Completa pode ser facilmente visualizada pela US, através da observação do eco endometrial hiperecóico, preenchido por imagens hipo-anecogênicas, irregulares, centrais ou margeando o miométrio, na ausência de embrião-feto. Além disso, é frequente o útero encontrar-se aumentado para a idade gestacional e os ovários apresentarem policistose (múltiplas formações císticas, de 4 a $8 \mathrm{~cm}$, anecogênicas, bem delimitadas, geralmente bilaterais). Nesses casos, $80 \%$ das MHC são diagnosticadas à US (BRAGA $\mathrm{A}$, et al., 2018). Já o diagnóstico da Mola Hidatiforme Parcial é mais tardio através do US, com alterações visíveis após a décima segunda semana de gestação, quando pode ser visualizado o feto apresentando malformações grosseiras, acompanhado de placenta com áreas hiperecogênicas tipo "flocos de neve". A US reconhecerá $90 \%$ das MHP com as alterações clássicas descritas (BRAGA A, et al., 2014).

Entretanto, cerca de $70 \%$ das molas parciais e 15\% das Molas Hidatiformes Completas somente são diagnosticadas pela avaliação histopatológica do material obtido por curetagem ou vácuo-aspiração, quando as características morfológicas das molas não estão bem desenvolvidas (ANDRADE JM, et al., 2009). É difícil realizar o diagnóstico diferencial entre MHC, MHP e aborto hidrópico com o uso apenas dos aspectos histológicos clássicos, sobretudo quando ocorre interrupção da gestação molar no primeiro trimestre (BRAGA A, et al., 2018).

Assim sendo atualmente também pode-se utilizar aliada ao exame histopatológico, como complementação diagnóstica, a imunocoloração para p57, um inibidor do ciclo celular. O gene p57KIP2 é transcrito pela mãe, mas o imprinting é determinado pelo pai, não ocorrendo expressão da proteína p57 nas molas completas, afastando a possibilidade de MHC (ALMEIDA LOG, et al., 2019).

\section{Tratamento da Doença Trofoblástica Gestacional}

Pacientes com diagnóstico de gravidez molar devem ser avaliados precocemente devido a potenciais complicações, como anemia e hipertireoidismo. Todas as pacientes deverão ser submetidas a um exame físico completo e exames laboratoriais, incluindo tipagem sanguínea e hematócrito, avaliação da tireóide, fígado e função renal (BERKOWITZ RS, GOLDSTEIN DP, 2009).

Os principais desafios para tratamento de pacientes com $\mathrm{MH}$ abrangem o diagnóstico precoce, por volta de 10-12 semanas de idade gestacional, esvaziamento uterino imediato e controle das complicações clínicas, seguimento pós-molar regular com dosagem sérica de hCG e contracepção eficiente no período de seguimento, uma vez que uma nova gestação poderia prejudicar o acompanhamento dos níveis de beta-hCG (MAESTÁ I e BRAGA A, 2012).

Após o diagnóstico de gravidez molar, deve-se pesquisar com cuidado a existência de complicações e, após a estabilização da paciente, deve-se escolher o método mais apropriado de evacuação. Na Mola Hidatiforme efetua-se o esvaziamento uterino, sendo o método de eleição a vácuo-aspiração, pois o risco de sangramento que sucede ao ato cirúrgico e de perfuração do útero são mínimos. A curetagem também pode ser realizada, mas com maiores riscos, sendo o principal o de perfuração uterina. O material eliminado é encaminhado para exame histopatológico, podendo trazer subsídios importantes com relação ao prognóstico (CORRÊA IB, et al., 2018). Em ambos os métodos de evacuação, devido ao risco aumentado de hipotensão e de edema agudo de pulmão, deve ser infundido ocitocina endovenosa (FERRAZ L, et al., 2015).

O uso de medicações como ocitócicos e análogos das prostaglandinas produzem contrações da musculatura uterina que, por elevarem a pressão intracavitária, podem causar embolização do tecido trofoblástico, não devendo ser utilizados em qualquer situação. Em casos específicos de mola hidatiforme parcial com presença fetal, o emprego cauteloso de agentes medicamentosos torna-se necessário para expulsão dos tecidos. A histerotomia, por estar relacionada a importante morbidade, tanto por permitir a passagem de tecido trofoblástico para a circulação, elevando o risco de NTG pós-molar, quanto por comprometer o futuro reprodutivo, é técnica proscrita (LIMA LLA, et al., 2017).

A histerectomia e a quimioterapia profilática também são opções terapêuticas. A histerectomia com a conservação dos anexos pode ser uma alternativa em mulheres com prole definida ou que não querem ter 
filhos. A histerectomia reduz o risco de neoplasia trofoblástica gestacional (NTG) pós-molar quando comparada ao esvaziamento uterino. Todavia, ainda há risco de NTG pós-molar em torno de 3-5\% (CORRÊA IB, et al., 2018). Este procedimento elimina o risco de invasão miometrial local, entretanto, ainda existe o risco de NTG metastática e, portanto, não permite a alta da paciente do seguimento pós-molar (FERRAZ L, et al., 2015).

A quimioterapia profilática, com Metrotrexate (MTX) ou com Actinomicina-D (Act-D), pode ser realizada em pacientes com preencham os critérios de alto-risco. São consideradas de alto risco pacientes com mola hidatiforme completa, altura uterina maior que a esperada para idade gestacional, $\beta$-hCG maior que 100.000 $\mathrm{mUI} / \mathrm{mL}$, presença de cisto ovariano teca luteínico com diâmetro maior que $6 \mathrm{~cm}$, idade superior a 35 a 40 anos. Reduzindo assim a progressão da doença para neoplasia trofoblástica gestacional, mas não é uma prática executada rotineiramente em nosso meio, além da evacuação uterina. (HAHN L, et al., 2018). Também deve ser incluído no tratamento, para todas as pacientes $\mathrm{RhD}$ negativas, imunoglobulina anti-D em doses habituais após o esvaziamento uterino, devido ao fato de o tecido trofoblástico expressar o fator RhD (BERKOWITZ RS e GOLDSTEIN DP, 2009).

\section{Segmento da Doença Trofoblástica Gestacional}

O segmento é feito para detectar precocemente os casos que apresentam persistência da doença. É relevante no seguimento pós-molar a periodicidade nas dosagens do hCG, que vai nos orientar sobre a evolução da doença trofoblástica, em um dos dois sentidos: resolução espontânea ou transformação maligna ( $15 \%$ a $40 \%$ das pacientes desenvolvem NTG pós-molar). Os títulos de hCG são mensurados semanalmente ou quinzenalmente. Se houver a normalização deste hormônio por três dosagens consecutivas, a avaliação passará a ser realizada mensalmente, durante seis meses. A neoplasia trofoblástica gestacional, segundo a FIGO, é caracterizada por quatro valores ou mais do $\mathrm{hCG}$ em platô, por pelo menos três semanas consecutivas $\left(1^{\circ}, 7^{\circ}, 14^{\circ}\right.$ e $21^{\circ}$ dias) ou elevação do hCG de $10 \%$ ou mais, por pelo menos duas semanas consecutivas. É essencial a adesão da paciente ao seguimento. Porém a dosagem de beta-hCG, não é o único componente do seguimento. Em pacientes com com valores do hCG acima do limite superior da curva de regressão normal, devem ser solicitados ultrassonografia pélvica com Doppler e radiografia de tórax (BRAGA A, et al., 2018). Além disso, em casos de sangramento anormal, mesmo com valores adequados de beta-hCG, também é solicitado US (CORRÊA IB, et al., 2018).

As pacientes devem ser orientadas sobre a importância da adesão a um método contraceptivo eficaz, uma vez que diante de uma gravidez, a elevação dos níveis de beta-hCG pode dificultar o diagnóstico de persistência ou malignização da doença. O uso de dispositivo intra-uterino não é indicado devido ao risco de perfuração uterina, sendo recomendado os contraceptivos orais e os injetáveis (HAHN L, et al., 2018).

A gravidez molar e a NTG provocam preocupação em relação às gestações subsequentes e fertilidade. Sabe-se que antecedente de $\mathrm{MH}$, aumenta em torno de $1 \%$ o risco de uma nova gestação molar, e 15 a $18 \%$ após duas MH. Devido ao maior risco de DTG, a avaliação ultrassonográfica é recomendada no primeiro trimestre para descartar possível DTG (BERKOWITZ RS e GOLDSTEIN DP, 2009).

\section{CONSIDERAÇÕES FINAIS}

A Doença Trofoblástica Gestacional é uma complicação obstétrica potencialmente grave. O seu diagnóstico e tratamento precoces são essenciais para que se obtenha o controle da doença. O diagnóstico definitivo é feito através da avaliação histopatológica, considerada padrão-ouro. $O$ tratamento visa a realização do esvaziamento da cavidade uterina, sendo o método de escolha a vácuo-aspiração. A histerectomia entra como método de eleição para mulheres com prole definida ou sem desejos de gestar, mas não elimina a necessidade da realização do segmento pós-molar. O segmento e acompanhamento das pacientes, através de dosagens seriadas do hormônio beta-hCG, são de extrema importância no que diz respeito à persistência da doença e à gestações subsequentes, pelo risco aumentado de uma nova gestação molar e de infertilidade. 


\section{REFERÊNCIAS}

1. DELMANTO LRMG, et al. A curva de regressão da gonadotrofina coriônica humana é útil no diagnóstico precoce da neoplasia trofoblástica gestacional pós-molar? Rev Bras Ginecol Obstet., 2007; 29(10):506-10.

2. RAMÍREZ CAG, et al. Factores de riesgo, diagnóstico histológico y concentraciones de beta-hCG en pacientes com mola hidatiforme. Med.Uis., 2018;31(1):39-46.

3. LÓPEZ CL, et al. Gestational Trophoblastic Neoplasia after Ectopic Molar Pregnancy: Clinical, Diagnostic, and Therapeutic Aspects. Rev Bras Ginecol Obstet, 2018;40:294-299.

4. ANDRADE JM, et al. Mola hidatiforme e doença trofoblástica gestacional. Rev Bras Ginecol Obstet., 2009; 31(2):94101.

5. MAESTÁ I, BRAGA A. Desafios do tratamento de pacientes com doença trofoblástica gestacional. Rev Bras Ginecol Obstet., 2012; 34(4):143-6.

6. SOARES BC, et al. A influência na determinação dos níveis do Hormônio Gonadotrofina Coriônica Humana (HCG) no monitoramento de neoplasias trofoblásticas gestacionais. Revista UNIABEU Belford Roxo, 2011; 4(7), Mar.-Ago.

7. LAVIE I, et al. Duration of human chorionic gonadotropin surveillance for partial hydatidiform moles. American Journal of Obstetrics and Gynecology, 2005; 192, 1362-4.

8. BRAGA A, et al. Doença trofoblástica gestacional - atualização. Revista HUPE, Rio de Janeiro, 2014;13(3):54-60.

9. CORRÊA IB, et al. Comorbidades associadas a mola hidatiforme: como diagnosticar e tratar. Revista de Patologia do Tocantins, 2018; 5(3): 68-74.

10. HAHN L, et al. Doença trofoblástica gestacional: diagnóstico e tratamento. Acta Medica, 2018; 39(2).

11. KERKMEIJER LGW, et al. Earlier Diagnosis and Serum Human Chorionic Gonadotropin Regression in Complete Hydatidiform Moles. Obstetrics \& Gynecology, 2009; 113(2), part 1.

12. BRAGA A, et al. Doença trofoblástica gestacional.São Paulo: Federação Brasileira das Associações de Ginecologia e Obstetrícia (Febrasgo); 2018. (Protocolo Febrasgo - Obstetrícia, no 23/Comissão Nacional Especializada em Doença Trofoblástica Gestacional).

13. BERKOWITZ RS, GOLDSTEIN DP. Molar Pregnancy. The New England Journal of Medicine, 2009; 360;16.

14. FERRAZ L, et al. Atualização no diagnóstico e tratamento da gravidez molar. JBM, 2015; 103(2).

15. ABREU EMF, et al. Mola hidatiforme. Rev Med Minas Gerais, 2009; 19(2 Supl 3): S14-S17.

16. TROMMEL NEV, et al. Diagnosis of hydatidiform mole and persistent trophoblastic disease: diagnostic accuracy of total human chorionic gonadotropin (hCG), free hCG \{alpha\}- and \{beta\}-subunits, and their ratios. European Journal of Endocrinology, 2005; 153(4): 565-575.

17. BISCARO A, et al. Frequência de mola hidatiforme em tecidos obtidos por curetagem uterina. Revista Brasileira de Ginecologia e Obstetrícia, 2012; 34(6): 254-8.

18. ALMEIDA LOG, et al. Mola hidatiforme parcial e completa: características clínicas e histológicas. Rev Med UFC, 2019; 59(4): 46-50.

19. LIMA LLA, et al. Papel da cirurgia no manejo de mulheres com doença trofoblástica gestacional. Rev. Col. Bras. Cir, 2017; 44(1): 94-101.

20. SUKSAY M, et al. Complete hydatidiform mole with co-existing fetus: Predictors of live birth. European Journal of Obstetrics \& Gynecology and Reproductive Biology, 2017; 212; 1-8. 